\title{
ARTICLE \\ Inhibition of Mcl-1 enhances cell death induced by the Bcl-2-selective inhibitor ABT-199 in acute myeloid leukemia cells
}

\author{
Daniel A Luedtke ${ }^{1,11}$, Xiaojia Niu 2,11, Yihang Pan ${ }^{3}$, Jianyun Zhao ${ }^{2,4}$, Shuang Liu ${ }^{2,4}$, Holly Edwards ${ }^{5,6}$, Kang Chen ${ }^{5,7,8}$, Hai Lin ${ }^{9}$, \\ Jeffrey W Taub ${ }^{4,6,10}$ and Yubin Ge $\mathrm{C}^{1,4,5,6}$
}

Acute myeloid leukemia (AML) is a serious disease. The 5 -year survival rates remain frustratingly low (65\% for children and $26 \%$ for adults). Resistance to frontline chemotherapy (usually cytarabine) often develops; therefore a new treatment modality is needed. $\mathrm{Bcl}-2$ family proteins play an important role in balancing cell survival and apoptosis. The antiapoptotic Bcl-2 family proteins have been found to be dysregulated in AML. ABT-199, a BH3 mimetic, was developed to target antiapoptotic protein Bcl-2. Although ABT-199 has demonstrated promising results, resistance occurs. Previous studies in AML show that ABT-199 alone decreases the association of proapoptotic protein Bim with $\mathrm{Bcl}-2$, but this is compensated by increased association of Bim with prosurvival protein Mcl-1, stabilizing Mcl-1, resulting in resistance to ABT-199. In this study, we investigated the antileukemic activity of the Mcl-1selective inhibitor A-1210477 in combination with ABT-199 in AML cells. We found that A-1210477 synergistically induced apoptosis with ABT-199 in AML cell lines and primary patient samples. The synergistic induction of apoptosis was decreased upon Bak, Bax and Bim knockdown. While A-1210477 treatment alone also increased Mcl-1 protein levels, combination with ABT-199 reduced binding of Bim to $\mathrm{Mcl}-1$. Our results demonstrate that sequestration of Bim by Mcl-1, a mechanism of ABT-199 resistance, can be abrogated by combined treatment with the Mcl-1 inhibitor A-1201477.

Signal Transduction and Targeted Therapy (2017) 2, e17012; doi:10.1038/sigtrans.2017.12; published online 7 April 2017

\section{INTRODUCTION}

Acute myeloid leukemia (AML) is a serious disease. In the United States, there are nearly twenty thousand new cases and over ten thousand deaths from this disease each year. ${ }^{1}$ The 5 -year survival rates, $65 \%$ for children and $26 \%$ for adults, remain frustratingly low. ${ }^{1}$ While changes in treatment and survival outcomes have proven successful in other leukemia subtypes like pediatric acute lymphoblastic leukemia and chronic myeloid leukemia, little has changed in the treatment of AML over the course of the last four decades. Resistance often develops against the main drug of treatment, cytarabine, thus a new treatment modality is urgently needed for this deadly disease.

The $\mathrm{Bcl}-2$ family of proteins plays a key role in balancing the decision between cell survival and apoptosis, and escape of apoptosis is a hallmark of cancer. ${ }^{2-4}$ This is especially important for the response to stress signals, including but not limited to cytotoxic agents like cytarabine and DNA damage. The $\mathrm{Bcl}-2$ family has been found to be dysregulated in AML, with increases in the antiapoptotic family members $\mathrm{BCl}-2, \mathrm{BCl}-\mathrm{xL}$ and $\mathrm{Mcl}-1$ having been found to play various roles. ${ }^{5,6}$ The $\mathrm{BH} 3$ mimetic ABT-199 was developed to target the antiapoptotic protein $\mathrm{BCl}-2$. $^{7,8}$
ABT-199 improved upon its predecessor ABT-263 by not targeting $\mathrm{BCl}-\mathrm{xL}$, whose inhibition leads to thrombocytopenia and limited clinical application. ${ }^{7}$ Results of a small phase I clinical trial have shown that ABT-199 has promising clinical activity, though a median time to relapse of 2.5 months indicates that resistance occurs quickly. 9,10 Thus, ABT-199 may be most effective when used in combination therapies.

Prosurvival $\mathrm{Bcl}-2$ family proteins, like $\mathrm{Bcl}-2$, sequester Bim to prevent Bim from inducing apoptosis. Bim interacts with Bax/Bak leading to Bax/Bak activation. Activated Bax/Bak form pores in the mitochondria outer membrane, leading to release of cytochrome $c$ and subsequent apoptosis. Targeting Bcl-2 with ABT-199 was expected to free Bim and induce apoptosis. Our previous studies in ABT-199-resistant AML cell lines and patients samples showed that ABT-199 decreased the association of Bim with Bcl-2 and increased the association of Bim with $\mathrm{Mcl}-1$ (which along with $\mathrm{Bcl}-$ $\mathrm{xL}$ also acts as a prosurvival Bcl-2 family protein in a similar fashion to $\mathrm{BCl}-2) .{ }^{11}$ In ABT-199-resistant cells, treatment with ABT-199 does not change the mitochondrial outer membrane permeabilization (MOMP), an event necessary for intrinsic apoptosis, pointing to changes in the balance of $\mathrm{Bcl}-2$ family members as the primary

\footnotetext{
${ }^{1}$ Cancer Biology Graduate Program, Wayne State University School of Medicine, Detroit, Michigan, USA; ${ }^{2}$ National Engineering Laboratory for AIDS Vaccine, School of Life Sciences, Jilin University, Changchun, China; ${ }^{3}$ Department of Pathology and Laboratory Medicine, Weill Cornell Medicine, New York, New York, USA; ${ }^{4}$ Department of Pediatrics, Wayne State University School of Medicine, Detroit, Michigan, USA; ${ }^{5}$ Department of Oncology, Wayne State University School of Medicine, Detroit, Michigan, USA; ${ }^{6}$ Molecular Therapeutics Program, Karmanos Cancer Institute, Wayne State University School of Medicine, Detroit, Michigan, USA; ${ }^{7}$ Department of Obstetrics and Gynecology, Wayne State University School of Medicine, Detroit, Michigan, USA; ${ }^{8}$ Mucosal Immunology Studies Team, National Institute of Allergy and Infectious Diseases, National Institutes of Health,

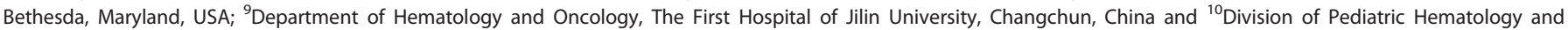
Oncology, Children's Hospital of Michigan, Detroit, Michigan, USA.
}

Correspondence: JW Taub (jtaub@med.wayne.edu) or Y Ge (gey@karmanos.org)

${ }^{11}$ These authors contributed equally to this work.

Received 5 January 2017; revised 22 February 2017; accepted 23 February 2017 
reason for resistance. ${ }^{11}$ On the basis of this, it is evident that both $\mathrm{BCl}-2$ and $\mathrm{Mcl}-1$ would have to be targeted to induce apoptosis in ABT-199-resistant AML cells.

Mcl-1 inhibition has evaded the current $\mathrm{Bcl}-2$ inhibitors, but recent advances have yielded $\mathrm{Mcl}-1$-specific inhibitors (such as A-1210477, a recently developed Mcl-1-specific inhibitor). ${ }^{12} \mathrm{Mcl}-1$ has been previously shown to decrease DNA damage and be necessary to inhibit Bak and Bax activation. ${ }^{13-16}$ The DNA damaging agent daunorubicin was found to decrease $\mathrm{Mcl}-1$ levels and synergize with ABT-199. ${ }^{11,17}$ As Mcl-1 is upregulated in resistant cells, the induced DNA damage may counteract this mode of resistance. In this study, we found that ABT-199 and A-1210477 synergistically induce apoptosis in ABT-199-resistant and ABT-199-sensitive AML cells. ABT-199 and A-1210477 combination treatment disrupts binding of Bim with $\mathrm{Bcl}-2$ and $\mathrm{Mcl}-1$. This synergy was not dependent on DNA damage and occurred through the $\mathrm{Bcl}-2$ family proteins.

\section{MATERIALS AND METHODS}

\section{Drugs}

ABT-199 and A-1210477 were purchased from Selleck Chemicals (Houston, TX, USA).

\section{Cell culture}

THP-1 and U937 cell lines were purchased from the American Type Culture Collection (Manassas, VA, USA). MOLM-13 cells were purchased from AddexBio (San Diego, CA, USA). The cell lines have not been authenticated since receiving them in our laboratory. The cell lines were cultured in RPMI 1640 media with 10\% fetal bovine serum (Life Technologies, Carlsbad, CA, USA) and $2 \mathrm{mM}$ L-glutamine, plus $100 \mathrm{U} \mathrm{ml}^{-1}$ penicillin and $100 \mu \mathrm{g} \mathrm{ml}^{-1}$ streptomycin, in a $37{ }^{\circ} \mathrm{C}$ humidified atmosphere containing $5 \% \mathrm{CO}_{2} / 95 \%$ air. Cell lines were tested for the presence of mycoplasma. Diagnostic AML blast samples derived from patients were purified by standard FicollHypaque density centrifugation, then cultured in RPMI 1640 with $20 \%$ fetal bovine serum, ITS Solution (Sigma-Aldrich, St Louis, MO, USA) and $20 \%$ supernatant of the 5637 bladder cancer cell line (as a source of granulocyte-macrophage colony-stimulating factor, granulocyte colonystimulating factor, interleukin-1 beta, macrophage colony-stimulating factor and stem cell factor). ${ }^{18-20}$

\section{Clinical samples}

Diagnostic AML blast samples were obtained from the First Hospital of Jilin University. Written informed consent was provided according to the Declaration of Helsinki. This study was approved by the Human Ethics Committee of The First Hospital of Jilin University. Clinical samples were screened for FLT3-ITD, NPM1, C-kit, CEBPA, IDH1, IDH2 and DNMT3A gene mutations and for fusion genes by real-time RT-PCR, as described previously. 18,21 Patient characteristics are listed in Table 1. Normal peripheral mononuclear cells were derived from healthy donors.

\section{Western blot analysis}

Cells were lysed in the presence of protease and phosphatase inhibitors (Roche Diagnostics, Indianapolis, IN, USA). Whole-cell lysates were subjected to SDS-polyacrylamide gel electrophoresis, electrophoretically transferred onto polyvinylidene difluoride (PVDF) membranes (Thermo Fisher Inc., Rockford, IL, USA) and immunoblotted with anti-Bcl-2 (ab692,
Abcam, Cambridge, MA, USA), -Bcl-xL (2764), -Mcl-1 (4572), -PARP (9542), -Bim (2819), - $\mathrm{H} 2 \mathrm{AX}$ (2577), -Bak (3814), -Bax (2774), -cleaved caspase-3 (9661, designated -cf caspase-3, Cell Signaling Technology, Danvers, MA, USA) or - $\beta$-actin (A2228, Sigma-Aldrich) antibody, as previously described. ${ }^{22,23} \mathrm{Imm}$ unoreactive proteins were visualized using the Odyssey Infrared Imaging System (Li-Cor, Lincoln, NE, USA), as described by the manufacturer. Western blots were repeated at least three times and one representative blot is shown. Densitometry measurements were made using Odyssey V3.0 (Li-Cor), normalized to $\beta$-actin, and calculated as the fold-change compared to the corresponding no drug treatment control.

Annexin V/PI staining and flow cytometry analysis

AML cells were treated with ABT-199 or A-1210477, alone or in combination, for 4 or $24 \mathrm{~h}$ and subjected to flow cytometry analysis using the annexin V-fluorescein isothiocyanate (FITC)/propidium iodide (PI) Apoptosis Kit (Beckman Coulter; Brea, CA, USA), as previously described. $^{24,25}$ Results are expressed as percent annexin $\mathrm{V}+$ cells. Experiments were performed three independent times in triplicate. For the AML cell lines, data presented are from one representative experiment, while patient sample experiments were performed once in triplicate due to limited sample size. Patient samples were chosen based on availability of adequate sample for the assay. The extent and direction of antileukemic interaction was determined by calculating the combination index (CI) values using CompuSyn software (Combosyn Inc., Paramus, NJ, USA). $\mathrm{Cl}<1, \mathrm{Cl}=1$ and $\mathrm{Cl}>1$ indicate synergistic, additive and antagonistic effects, respectively. ${ }^{24,26}$

\section{Immunoprecipitation}

AML cell lines were treated for $4 \mathrm{~h}$ and then the cells were lysed using $1 \%$ CHAPS, $5 \mathrm{~mm} \mathrm{MgCl} 2,150 \mathrm{~mm} \mathrm{NaCl}, 1 \mathrm{~mm}$ EDTA, 1 mm EGTA, 20 mm Tris and $0.05 \%$ Tween-20 in the presence of protease inhibitors. Immunoprecipitation of Bim and $\mathrm{Mcl}-1$ was performed as previously described ${ }^{27}$ using $2 \mu \mathrm{g}$ of anti-Bim (2819, Cell Signaling Technology) or anti-Mcl-1 (SC-819, Santa Cruz Biotechnology, Santa Cruz, CA, USA) antibody, 1 mg protein lysate, and Protein $A$ agarose beads (Roche Diagnostics). Proteins were eluted using $50 \mathrm{mM}$ glycine, $\mathrm{pH}$ 2.0, and then analyzed by Western blotting.

\section{shRNA knockdown}

The pMD-VSV-G and delta 8.2 plasmids were gifts from Dr Dong at Tulane University. Bim and non-target control (NTC) shRNA lentiviral vectors were purchased from Aldrich. Lentivirus production and transduction were carried out as previously described. ${ }^{28}$ Briefly, TLA-HEK293T cells were transfected with pMD-VSV-G, delta 8.2, and lentiviral shRNA constructs using Lipofectamine and Plus reagents (Life Technologies) according to the manufacturer's instructions. Virus containing culture medium was harvested $48 \mathrm{~h}$ post transfection. Cells were transduced overnight using $1 \mathrm{ml}$ of virus supernatant and $4 \mu \mathrm{g}$ of polybrene and then cultured for an additional $48 \mathrm{~h}$ prior to selection with puromycin.

\section{Alkaline comet assay}

U937 cells were treated for $4 \mathrm{~h}$ with ABT-199 and/or A-1210477 and subjected to alkaline comet assay as previously described. ${ }^{28}$ Slides were stained with SYBR Gold (Life Technologies), and then imaged on an Olympus BX-40 microscope equipped with a DP72 microscope camera and Olympus cellSens Dimension software (Olympus America Inc., Center Valley, PA, USA). Approximately 50 comets per gel were scored using CometScore (TriTek Corp, Sumerduck, VA, USA).

Table 1. Patient characteristics for primary AML patient samples

\begin{tabular}{llllllll}
\hline Patient & Gender & Age (years) & Disease Status & FAB subtype & Cytogenetics & Blast purity (\%) & Gene mutation \\
\hline AML\#110 & Female & 66 & Relapsed & M4 & $45, \mathrm{XX},-7$ & 48.0 & \\
AML\#111 & Male & 58 & Newly diagnosed & M2 & $46, X Y$ & 63.5 & CEBP $\alpha$ double mutation, DNMT3A mutation, \\
AML\#112 & Male & 46 & Newly diagnosed & M1 & $46, X Y$ & 92.0 & GATA2 mutation \\
AML\#113 & Female & 10 & Newly diagnosed & M5 & $46, X X$ & 97.0 & \\
\hline \multicolumn{7}{ll}{ Abbreviation: AML, acute myeloid leukemia. }
\end{tabular}




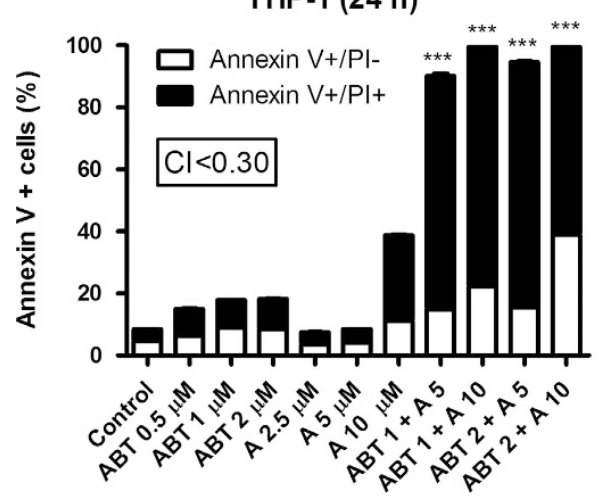

C

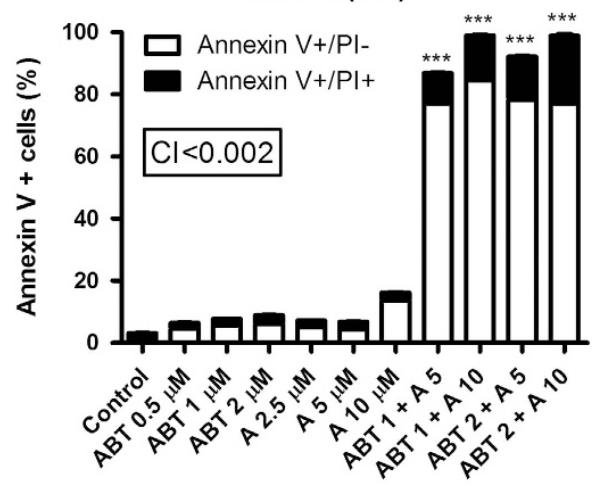

e

THP-1 (4 h)

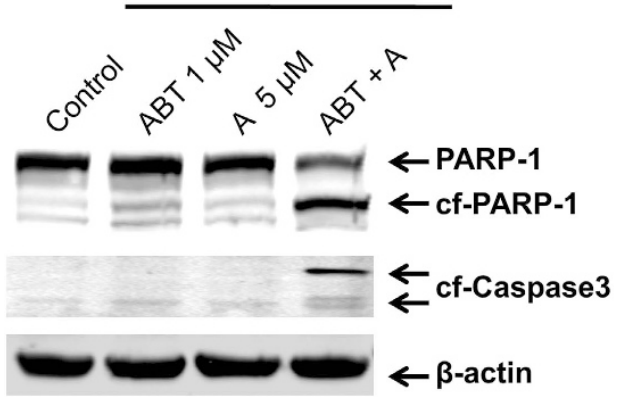

g

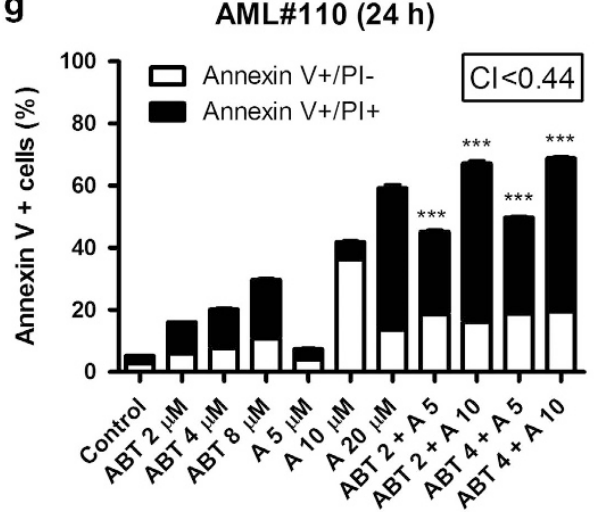

b

U937 (24 h)

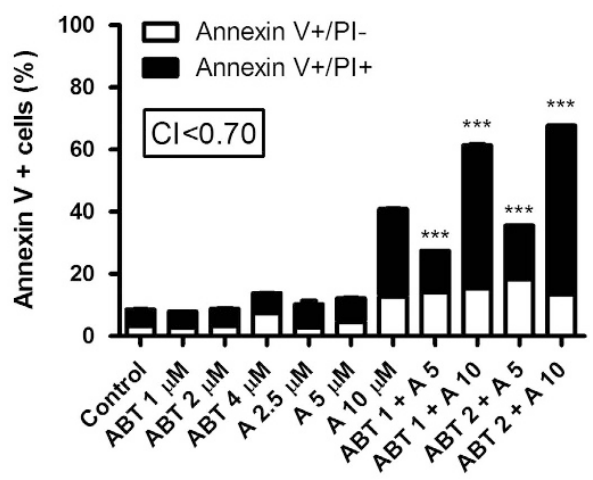

d

U937 (4 h)

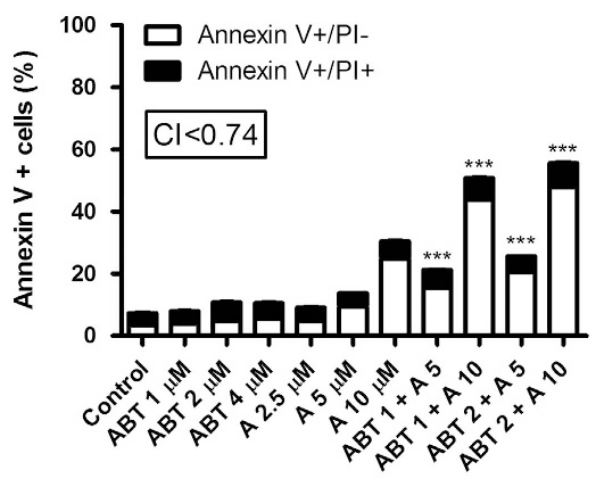

f

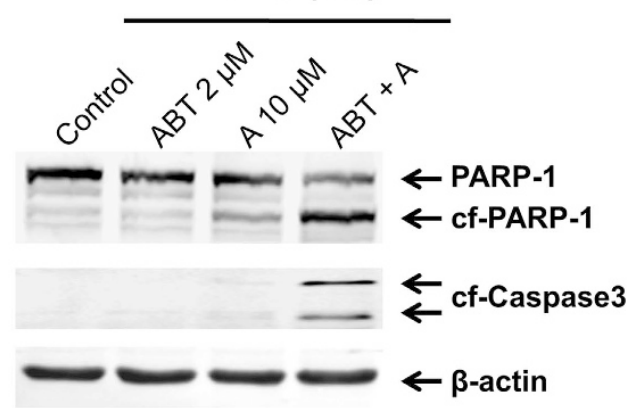

h

AML\#110 (24 h)

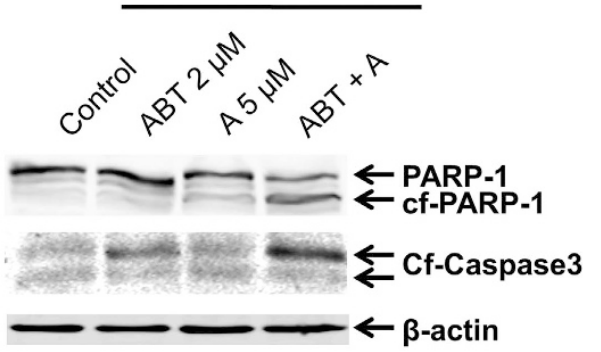

Figure 1. A-1210477 synergizes with ABT-199 to induce apoptosis in ABT-199-resistant acute myeloid leukemia (AML) cells. (a-d) THP-1 and U937 cells were treated with ABT-199 and A-1210477, alone or in combination, for 4 or $24 \mathrm{~h}$ and then subjected to annexin V/PI staining and flow cytometry analyses. ${ }^{* * *} P<0.001$. Combination index $(\mathrm{Cl})$ values were calculated using CompuSyn software. (e, f) THP-1 and U937 cells were treated with ABT-199 or A-1210477 alone or in combination for $4 \mathrm{~h}$. Whole-cell lysates were subjected to Western blotting and probed with the indicated antibodies. (g, h) Primary AML patient sample AML\#110 was treated with ABT-199 and A-1210477, alone or in combination, for $24 \mathrm{~h}$ and then subjected to annexin V/PI staining and flow cytometry analyses (g). Whole-cell lysates were subjected to Western blotting and probed with the indicated antibodies (h). ${ }^{* *} P<0.001$. Cl values were calculated using CompuSyn software. 
Statistical analysis

Differences were compared using the two-sample $t$-test. Statistical analyses were performed with GraphPad Prism 5.0. Error bars represent \pm s.e.m. The level of significance was set at $P<0.05$.

\section{RESULTS}

A-1210477 synergizes with ABT-199 in ABT-199-resistant AML cells To test our hypothesis that A-1210477 (abbreviated A) can synergize with ABT-199 (abbreviated ABT) to induce apoptosis, we tested various concentrations of ABT-199 and A-1210477 alone and in combination in ABT-199-resistant (U937, THP-1, and a primary $A M L$ patient sample derived at relapse) $A M L$ cells. The combination index $(\mathrm{Cl})$ was used to determine synergy. $\mathrm{Cl}=1$ denotes an additive effect while $\mathrm{Cl}<0.9$ denotes synergy, and $\mathrm{Cl}<0.3$ denotes strong synergy. ${ }^{26}$ At $24 \mathrm{~h}$, synergy was observed between the two drugs for THP-1 $(\mathrm{Cl}<0.30)$ and U937 $(\mathrm{Cl}<0.70)$ cell lines (Figures $1 \mathrm{a}$ and $\mathrm{b}$ ). Annexin $\mathrm{V}$ positive cells were largely propidium iodide $(\mathrm{PI})$ positive as well, indicating that the cells were late apoptotic or necrotic. ${ }^{29}$ Annexin V/PI positivity was assessed after $4 \mathrm{~h}$ treatment to determine if cells may have undergone apoptosis. At $4 \mathrm{~h}$, synergy was still observed for THP-1 $(\mathrm{Cl}<0.002)$ and $\mathrm{U} 937(\mathrm{Cl}<0.74)$ cell lines and a majority of the Annexin V positive cells were PI negative, indicating that the cells underwent apoptosis (Figures 1c and d). Corroborating this, cleavage of PARP and caspase- 3 was strongly enhanced in the combination treatment when compared to ABT-199 or A-1210477 alone in THP-1 and U937 cells (Figures 1e and f). Similar results were achieved in a primary patient sample ex vivo (Figures $1 \mathrm{~g}$ and $\mathrm{h}$ ). In summary, A-1210477 is able to synergize with ABT-199 to induce apoptosis in otherwise ABT-199-resistant AML cells in a synergistic manner.

A-1210477 treatment increases $\mathrm{Mcl}-1$ protein levels but releases Bim from Mcl-1

Having observed the synergy of ABT-199 and A-1210477, the next question was to determine how treatment affected levels of relevant $\mathrm{BCl}-2$ proteins and their interactions. Protein levels of $\mathrm{BCl}-2, \mathrm{Mcl}-1, \mathrm{BCl}-\mathrm{xL}$, and Bim were determined by Western blotting. Individual treatment with ABT-199 and A-1210477 caused increased levels of $\mathrm{Mcl}-1$ without changing the levels of $\mathrm{Bcl}-2$, Bim, and Bcl-xL in THP-1, U937, and a primary AML patient sample derived at relapse (Figures $2 \mathrm{a}-\mathrm{C}$ ). Combination treatment appeared to decrease $\mathrm{Mcl}-1$ levels compared to A-1210477 alone, though levels remained similar to or higher than vehicle control treated cells, suggesting that disruption of the interaction of $\mathrm{Mcl}-1$ with $\mathrm{Bcl}-2$ family proteins played a critical role in the synergistic effects. To determine if interactions of $\mathrm{Bcl}-2$ family members with Bim were disrupted by drug treatments, co-immunoprecipitation was performed. A-1210477 disrupted the interaction between Bim
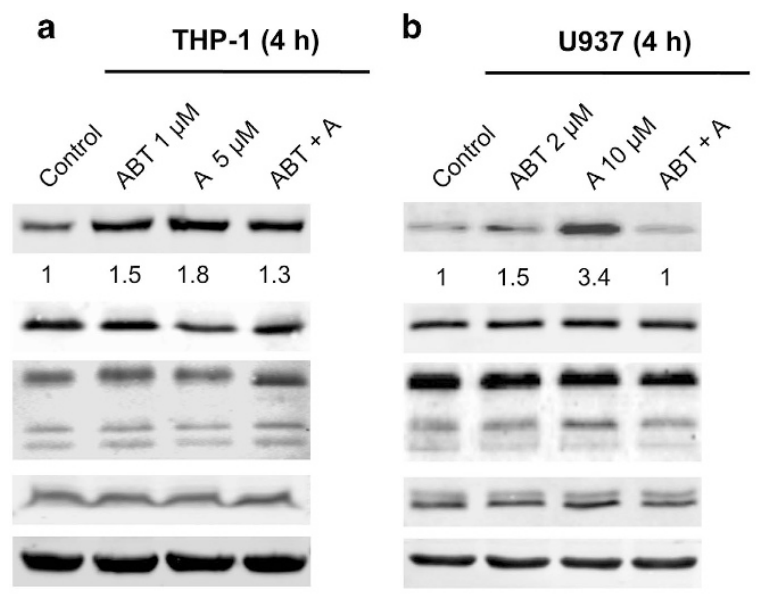

d

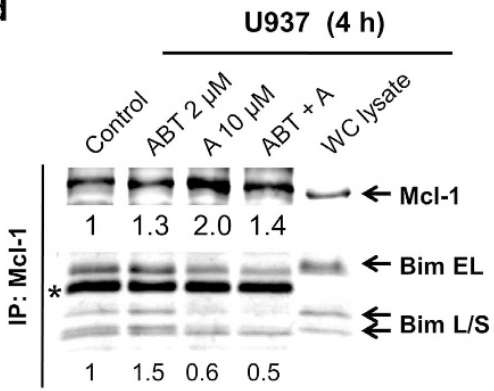

C

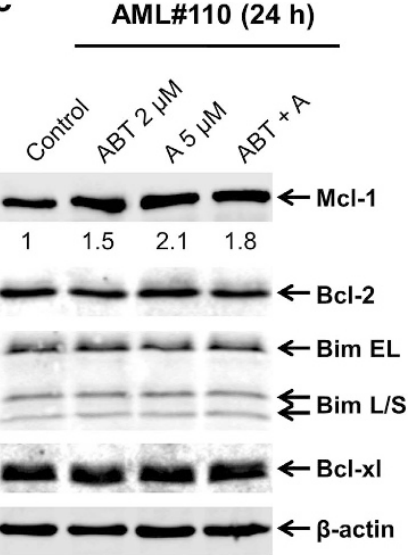

U937 (4 h)

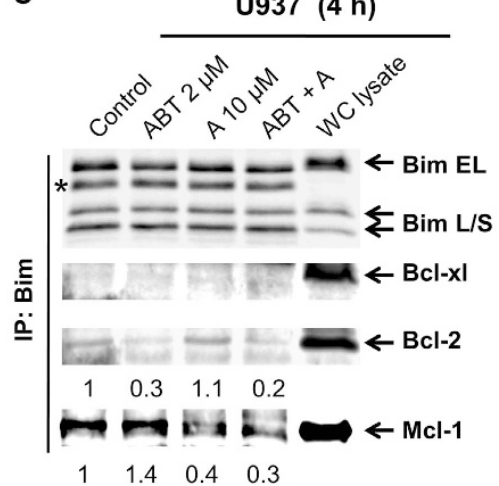

Figure 2. A-1210477 treatment increases Mcl-1 protein levels but releases Bim from Mcl-1. (a-c) THP-1, U937, and primary acute myeloid leukemia (AML) patient sample cells (AML\#110) were treated with ABT-199 and A-1210477, alone or in combination, for $4 \mathrm{~h}$ or $24 \mathrm{~h}$. Whole-cell lysates were subjected to western blotting and probed with the indicated antibodies. Relative densitometry measurements of Mcl- 1 expression were measured using Odyssey Software V3.0. (d, e) U937 cells were treated with ABT-199 and A-1210477, alone or in combination, for $4 \mathrm{~h}$. Mcl-1 (d) or Bim (e) was immunoprecipitated from whole-cell lysates and then subjected to western blotting and probed with the indicated antibodies. Relative densitometry measurements of Mcl-1, Bim and Bcl-2 were measured using Odyssey Software V3.0. ${ }^{\prime * \prime}$ indicates the light chain of the anti-Mcl-1 or -Bim antibody. 
and $\mathrm{Mcl}-1$ (Figure 2d), which surprisingly occurred despite the presence of elevated $\mathrm{Mcl}-1$. The binding of Bim with $\mathrm{BCl}-2$ was disrupted by ABT-199 and not A-1210477 (Figure 2e). The binding of Bim with $\mathrm{BCl}-2$ and $\mathrm{Mcl}-1$ was disrupted by the combination treatment. $\mathrm{BCl}-\mathrm{xL}$, another antiapoptotic $\mathrm{Bcl}-2$ family member which binds to Bim, did not compensate for this disruption, indicating that Bim was possibly unbound allowing for it to carry out its proapoptotic role.
The effect of Bim, Bax and Bak knockdown on apoptosis induced by ABT-199 and A-1210477 in AML cells

To confirm the contribution of Bim to apoptosis induced by ABT-199 and A-1210477 combined treatment, shRNA knockdown was performed. Knockdown of Bim in U937 and THP-1 (Figures 3a and c) significantly reduced apoptosis in response to A-1210477 and combined treatment in both cell lines (Figures $3 \mathrm{~b}$ and d). Bim knockdown also significantly reduced apoptosis induced by a

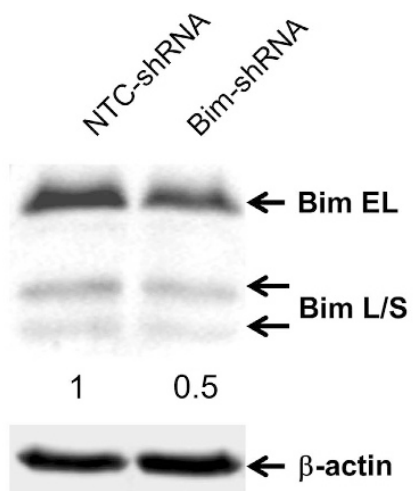

C

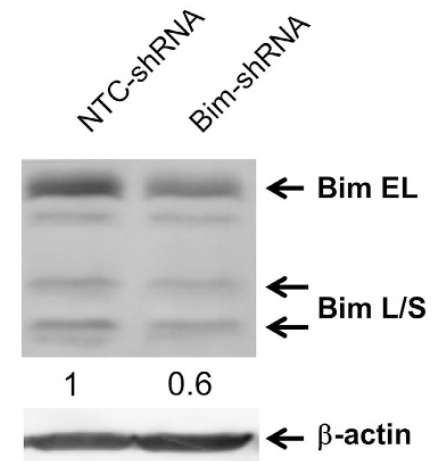

e

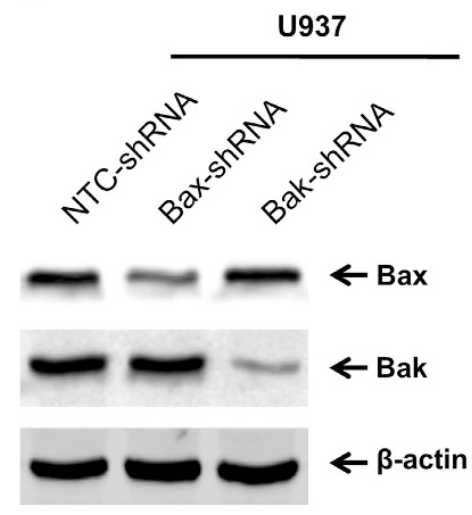

b

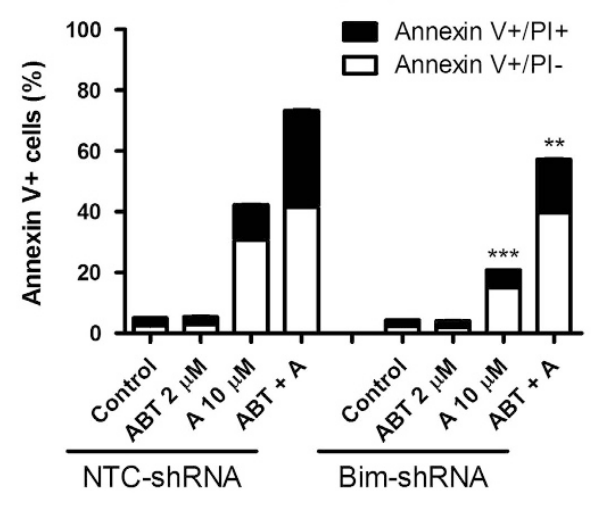

d

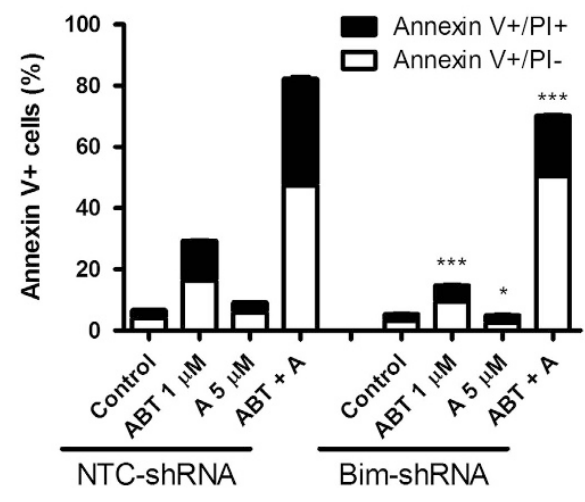

f

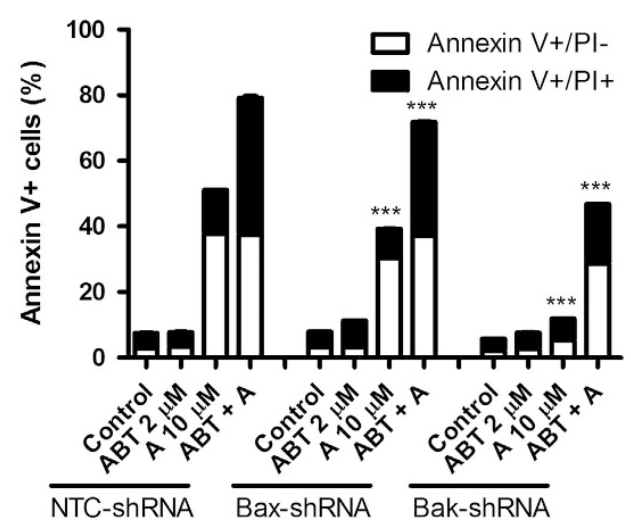

Figure 3. The effect of Bim, Bax and Bak knockdown on apoptosis induced by ABT-199 and A-1210477 in acute myeloid leukemia (AML) cell lines. (a, c) U937 (a) or THP-1 (c) cells were infected with non-template control (NTC-shRNA) or Bim (Bim-shRNA) shRNA lentivirus. Whole-cell lysates were subjected to western blotting and probed with the indicated antibodies to confirm the knockdown. (b, d) U937 (b) or THP-1 (d) shRNA knockdown cells were treated with ABT-199 and A-1210477, alone or in combination, for $4 \mathrm{~h}$ and then subjected to annexin V/PI staining and flow cytometry analyses. ${ }^{*} P<0.05$, ${ }^{* *} P<0.01$ and ${ }^{* *} P<0.001$. (e) U937 cells were infected with non-template control (NTC-shRNA), Bax (Bax-shRNA) or Bak (Bak-shRNA) shRNA lentivirus. Whole-cell lysates were subjected to western blotting and probed with the indicated antibodies to confirm the knockdowns. (f) The U937 Bax and Bak knockdown cells were treated with ABT-199 and A-1210477, alone or in combination, for $4 \mathrm{~h}$ and then subjected to annexin V/PI staining and flow cytometry analyses. ${ }^{* * *} P<0.001$. 


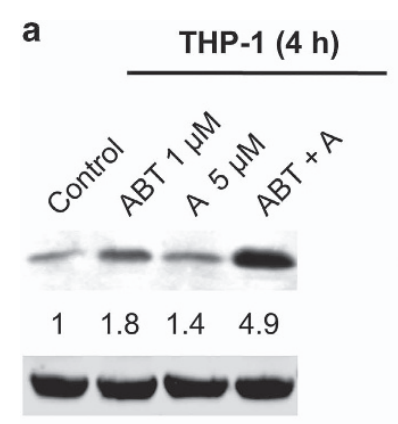

b
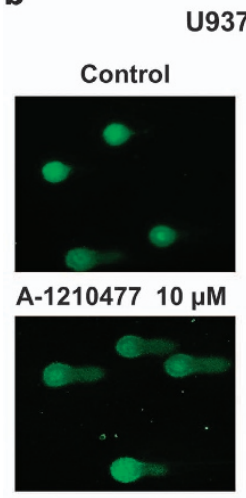

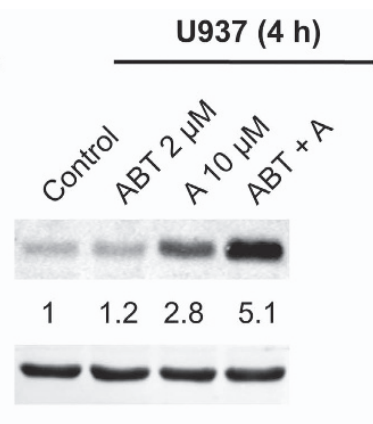

C
AML\#110 (24 h)

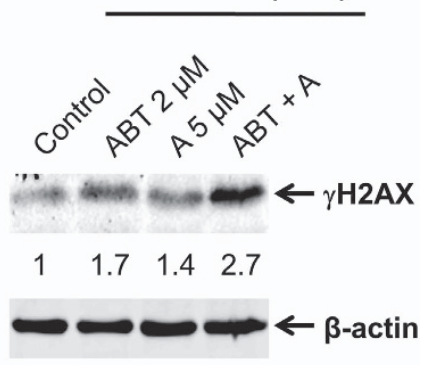

937 (4 h)

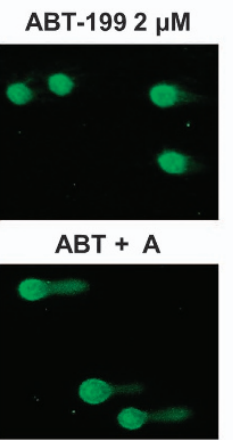

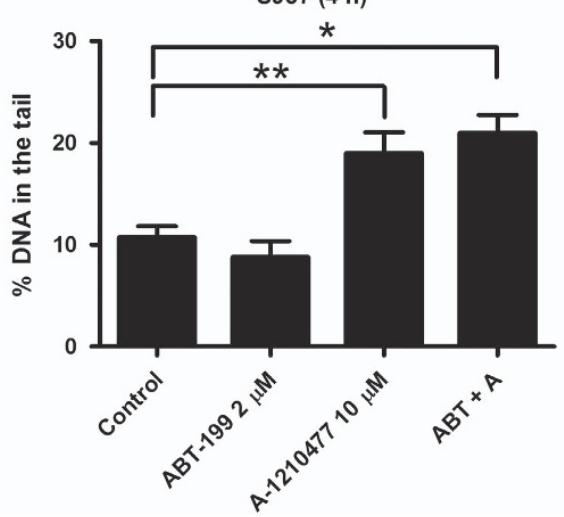

Figure 4. A-1210477 induces DNA damage in acute myeloid leukemia (AML) cells. (a) THP-1, U937, and a primary AML patient sample cells (AML\#110) were treated with ABT-199 and A-1210477, alone or in combination, for $4 \mathrm{~h}$ or $24 \mathrm{~h}$. Whole-cell lysates were subjected to Western blotting and probed with the indicated antibody. Relative densitometry measurements of $\gamma \mathrm{H} 2 \mathrm{AX}$ expression were measured using Odyssey Software V3.0. (b) U937 cells were treated with ABT-199 and A-1210477, alone or in combination, for $4 \mathrm{~h}$ and then subjected to alkaline comet analyses. Representative images are shown. (c) Alkaline comet assay results are graphed as median percent DNA in the tail from four replicate gels \pm s.e.m. ${ }^{*} P<0.05$ and ${ }^{* *} P<0.01$.

ABT-199 in THP-1 cells (Figure 3d). Bak and Bax knockdowns were performed in U937 cells due to the critical roles they play in the intrinsic apoptotic pathway (Figure 3e). As expected, knockdown of Bax or Bak (especially Bak) also reduced apoptosis significantly in the A-1210477 and combined treatments (Figure 3f). These results demonstrate that the canonical pathway of intrinsic apoptosis using Bim, Bak and Bax plays an important role in induction of apoptosis in response to A-1210477 treatment alone or in combination with ABT-199.

\section{A-1210477 induces DNA damage in AML cells}

Mcl-1 has a known role in both apoptosis and the DNA damage response. We previously demonstrated that ABT-199 treatment enhances DNA damage induced by DNA damaging agents, ${ }^{11,30}$ thus the question remained whether the synergistic action of A-1210477 combined with ABT-199 was due to apoptosis and/or DNA damage. $\mathrm{Y}-\mathrm{H} 2 \mathrm{AX}$, a surrogate marker for DNA damage was measured by western blot. ${ }^{31}$ The level of $\mathrm{Y}-\mathrm{H} 2 \mathrm{AX}$ increased following ABT-199 or A-1210477 treatment, and increased after combined treatment in THP-1, U937, and a patient sample (Figure 4a). However $\mathrm{\gamma}-\mathrm{H} 2 \mathrm{AX}$ levels can indicate late apoptosis as well. To further elucidate the extent of DNA damage, the comet assay was performed. DNA damage, as measured by alkaline comet assay (Figures $4 \mathrm{~b}$ and $\mathrm{C}$ ), increased dramatically with A-1210477 treatment but not ABT-199 treatment. Interestingly, combination treatment did not increase DNA damage compared to A-1210477 treatment. While A-1210477 may induce some apoptosis by DNA damage, its synergistic interaction with ABT-199 is not due to DNA damage and is likely due to the apoptotic function of Mcl-1.
A-1210477 synergizes with ABT-199 in ABT-199-sensitive AML cells To further increase the clinical relevance of the study, A-1210477 and ABT-199 combination treatment was performed in ABT-199sensitive MOLM-13 cells, newly diagnosed AML patient samples, and normal donor peripheral blood mononuclear cells (PMNCs). Treatment of MOLM-13 with ABT-199 and A-1210477 synergistically induced apoptosis (Figure $5 \mathrm{a}, \mathrm{Cl}<0.16$ ), accompanied by caspase- 3 and PARP cleavage (Figure $5 b$ ). Similar to resistant cell lines, $\mathrm{BCl}-2$ and Bim levels were unchanged and $\mathrm{Mcl}-1$ was induced by A-1210477 treatment alone (Figure 5c). However, Mcl1 was not detected after combined treatment. Treatment of 3 newly diagnosed AML patient samples with A-1210477 and ABT-199 synergistically induced apoptosis, as well $(\mathrm{Cl}<0.40$, $\mathrm{Cl}<0.49, \mathrm{Cl}<0.05$, respectively, Figure $5 \mathrm{~d}$ ). In several relatively ABT-199-sensitive and -resistant AML cell lines and patient samples, ABT-199 and A-1210477 combination treatment synergistically induced apoptosis, suggesting that this combination may work regardless of ABT-199 sensitivity.

Lastly, to test the effects of combined treatment on normal cells in addition to AML cells, 5 normal donor peripheral blood mononuclear cells were subjected to single drug treatment of either ABT-199 or A-1210477 to determine $I C_{50}$ values. ABT-199 IC $C_{50}$ values ranged from 5.5 to $48.3 \mu \mathrm{m}$, while A-1210477 IC 50 values ranged from 3.3 to $7.1 \mu \mathrm{m}$ (Figure $5 e$ ). $I C_{50}$ values were also determined for $A-1210477$ in the presence of set concentrations of ABT-199. Surprisingly, it was found that A-1210477 and ABT-199 act synergistically to inhibit cell proliferation in normal PMNCs (Figure 5f).

\section{DISCUSSION}

It is widely believed that leukemic stem cells which have escaped chemotherapy treatment contribute to AML relapse. ${ }^{32}$ Although it 
has been shown that leukemic stem cells overexpress $\mathrm{BCl}-2^{33}$ and that the $\mathrm{BCl}$-2-selective inhibitor ABT-199 has shown promise, leading to clinical trials in $A M L,{ }^{34}$ resistance remains an issue. A phase I clinical trial showed a $19 \%$ complete response rate for ABT-199 monotherapy. ${ }^{9}$ However, all of the patients relapsed within a short period of time (median time to relapse was only
2.5 months), suggesting that ABT-199 would best be used in combination regimens. ${ }^{9,10}$ In this study, we demonstrated that inhibition of $\mathrm{Mcl}-1$ enhanced ABT-199-induced apoptosis in both ABT-199-sensitive and ABT-199-resistant AML cells.

Previously, we reported that the Chk1 inhibitor LY2603618 induces DNA damage, decreases Mcl-1 levels (even in the a

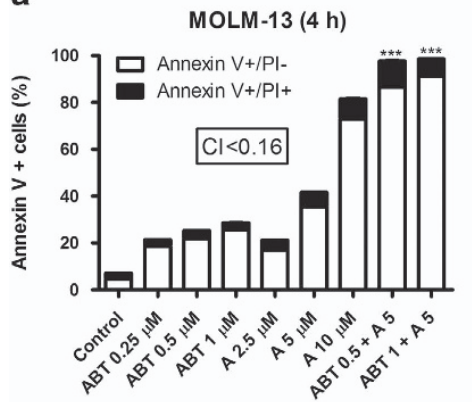

d

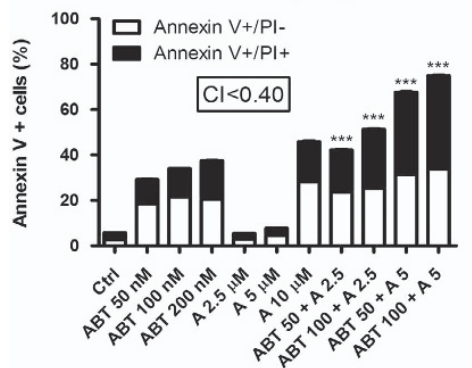

e

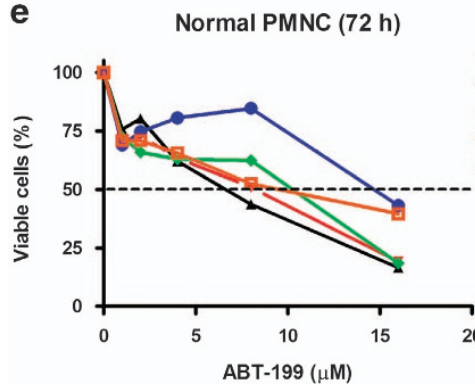

b

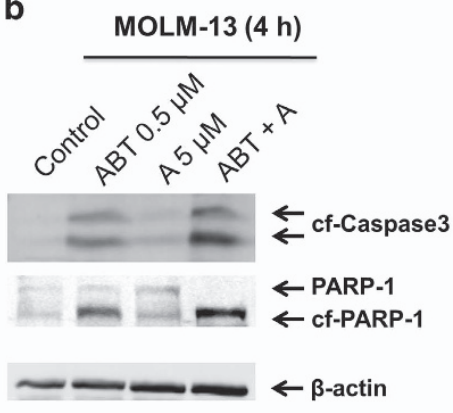

C
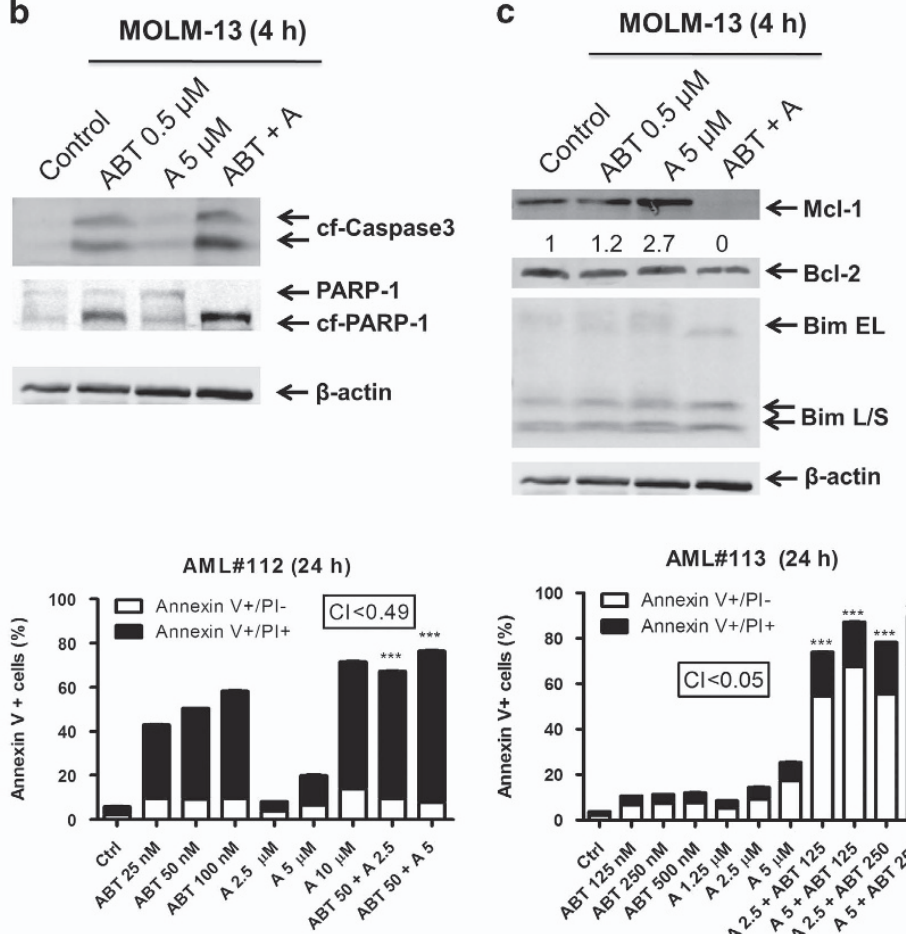

AML\#113 (24 h)

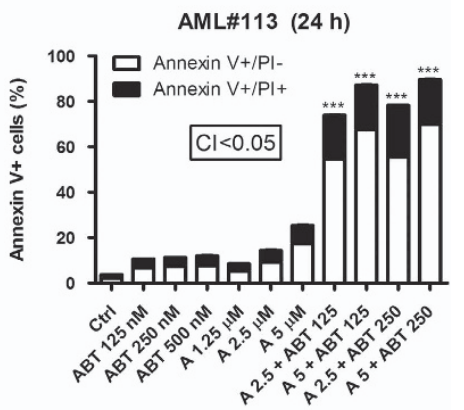

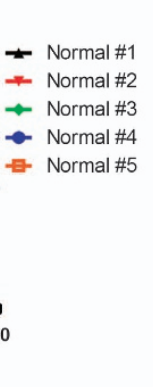

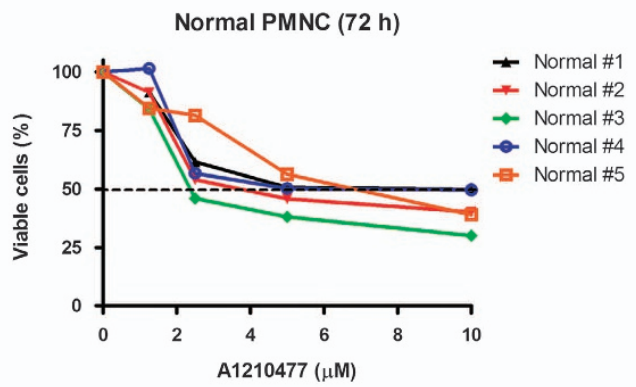

f
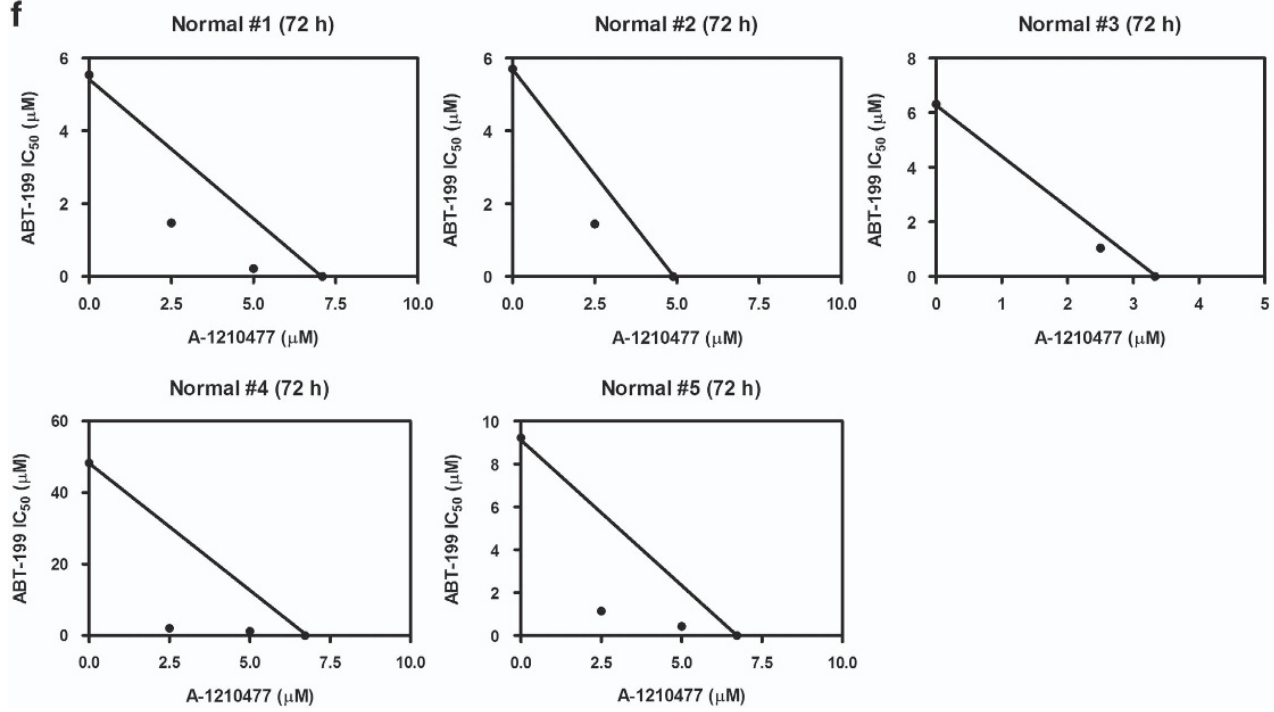
Figure 5. A-1210477 synergizes with ABT-199 in ABT-199-sensitive acute myeloid leukemia (AML) cells. (a) MOLM-13 cells were treated with ABT-199 and A-1210477, alone or in combination, for $4 \mathrm{~h}$ and then subjected to annexin V/PI staining and flow cytometry analyses. ${ }^{* * *} P<0.001$. Combination index $(\mathrm{Cl})$ values were calculated using CompuSyn software. $(\mathbf{b}, \mathbf{c})$ MOLM-13 cells were treated with ABT-199 and A-1210477, alone or in combination, for $4 \mathrm{~h}$. Whole-cell lysates were subjected to western blotting and probed with the indicated antibodies. (d) Primary AML patient sample cells (AML\#111-113) were treated with ABT-199 and A-1210477, alone or in combination, for $24 \mathrm{~h}$ and then subjected to annexin V/PI staining and flow cytometry analyses. ${ }^{* * *} P<0.001$. Cl values were calculated using CompuSyn software. (e, f) MTT analyses were performed on normal peripheral blood mononuclear cells (PMNCs) to determine proliferation inhibition at the indicated concentrations of A-1210477 and ABT-199, alone or in combination. Patient sample data are means of duplicates due to limited sample. Standard isobologram analyses of drug interactions were performed to determine the extent and direction of the drug interactions. The $\mathrm{IC}_{50}$ values of each drug are plotted on the axes; the solid line represents the additive effect, while the points represent the concentrations of each drug resulting in $50 \%$ inhibition of proliferation. Points falling below the line indicate synergism whereas those above the line indicate antagonism.

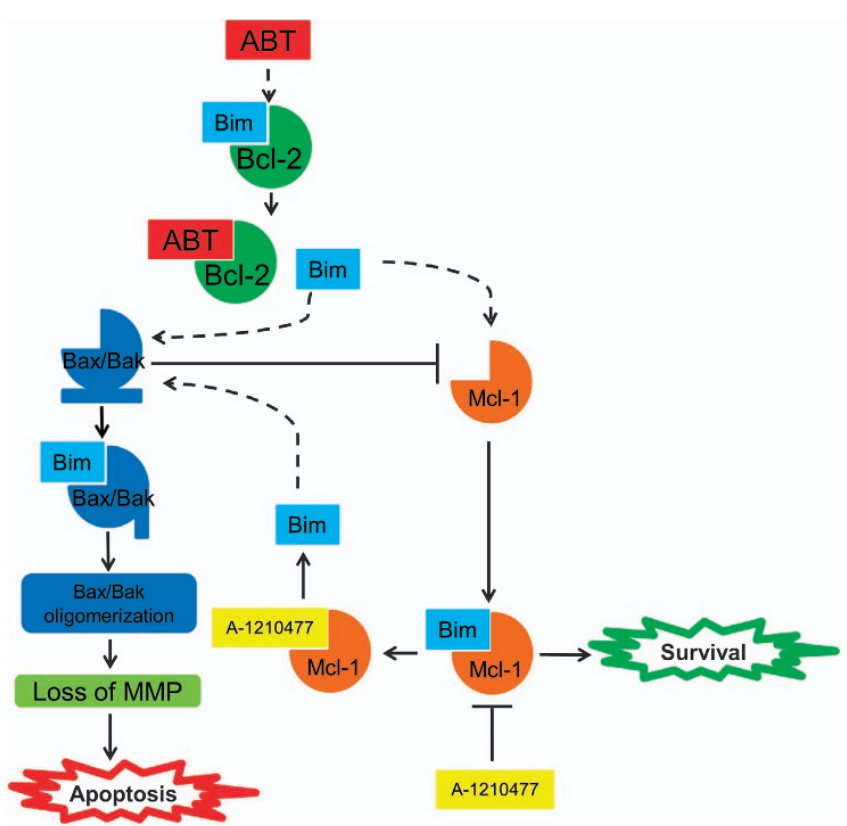

Figure 6. Proposed mechanism for ABT-199 in combination with A-1210477 in AML cells. ABT-199 treatment releases Bim from Bcl-2. In ABT-199-sensitive cells, there is an inadequate amount of $\mathrm{Mcl}-1$ to sequester all of the released Bim, resulting in free Bim, which can then activate Bak/Bax, leading to apoptosis. In ABT-199-resistant cells, the Bim released from $\mathrm{BCl}-2$ is sequestered by $\mathrm{Mcl}-1$, leading to stabilization of $\mathrm{Mcl}-1$, and ultimately resulting in survival. In the combined drug treatment, addition of A-1210477 abolishes sequestration of Bim by Mcl-1, allowing Bim to activate Bax/Bak, resulting in enhanced apoptosis.

presence of ABT-199), and synergistically induces apoptosis with ABT-199. ${ }^{30}$ In addition, we have also shown that DNA damage induced by chemotherapeutic agents is enhanced by ABT-199. ${ }^{11,17}$ While A-1210477 did cause DNA damage, enhanced DNA damage was not observed in the combined treatment, suggesting that DNA damage may not have played a role in the synergistic induction of apoptosis by the two agents in AML cells. On the basis of our current and previous findings, we propose the following mechanism of action of ABT-199 in combination with A-1210477. ABT-199 treatment releases Bim from $\mathrm{Bcl}-2$. In sensitive cells, it is possible that there is not enough $\mathrm{Mcl}-1$ to sequester all of the released Bim, resulting in free Bim, which can then activate Bax/Bak leading to apoptosis. In contrast, the Bim released from $\mathrm{BCl}-2$ is sequestered by $\mathrm{Mcl}-1$, stabilizing $\mathrm{Mcl}-1$ and ultimately resulting in survival in the ABT-199-resistant cells. ${ }^{11}$ Addition of A-1210477 reduces $\mathrm{Mcl}-1$ interaction with Bim, decreasing sequestration of Bim, allowing for activation of Bax/ Bak, and eventually resulting in apoptosis (Figure 6).
Although simultaneously inhibiting $\mathrm{BCl}-2$ and $\mathrm{Mcl}-1$ results in synergistic induction of apoptosis in AML cells, the treatment also synergistically inhibited proliferation of normal PMNCs, indicating that toxicity may be a concern moving forward. While direct inhibition of $\mathrm{Mcl}-1$ may be toxic, indirect targeting of Mcl-1 may prove to be more fruitful. We have previously demonstrated that DNA damaging agents daunorubicin, cytarabine, and CHK1-selective inhibitor LY2603618 downregulate Mcl1 and enhance ABT-199-induced apoptosis in AML cells. ${ }^{11,30}$ It has been reported that dual PI3K/mTOR inhibitor, NVP-BEZ235, downregulates $\mathrm{Mcl}-1$ and sensitizes leukemic cells to ABT-199. ${ }^{35}$ Inhibition of CDK9 has also been shown to enhance ABT-199 sensitivity through downregulation of $\mathrm{Mcl}-1 .^{36}$ In summary, the Mcl-1 inhibitor A-1210477 enhances ABT-199-induced apoptosis in AML cells, though synergistic results were also revealed in normal PMNCs. These results warrant pursuing new $\mathrm{Mcl}-1$ inhibitors or therapies, which indirectly inhibit Mcl-1 for combination with ABT-199 in AML.

\section{ACKNOWLEDGEMENTS}

This study was supported by grants from the National Natural Science Foundation of China, NSFC 31671438 and NSFC 31471295, the Graduate Innovation Fund of Jilin University, Hyundai Hope On Wheels, the Ring Screw Textron Endowed Chair for Pediatric Cancer Research, Children's Hospital of Michigan Foundation, Kids Without Cancer, Lafontaine Family/U Can-Cer Vive Foundation, the Decerchio/Guisewite Family, Justin's Gift, Elana Fund, and the Ginopolis/Karmanos Endowment and supported by Jilin University, Changchun, China, Wayne State University School of Medicine, the China Scholarship Council, and the Barbara Ann Karmanos Cancer Institute.

\section{DISCLAIMER}

The funders had no role in the study design, data collection, analysis and interpretation of the data, decision to publish or preparation of the manuscript.

\section{COMPETING INTEREST}

The authors declare no conflict of interest.

\section{REFERENCES}

1 Siegel RL, Miller KD, Jemal A. Cancer statistics, 2016. CA Cancer J Clin 2016; 66: 7-30.

2 Adams JM, Cory S. The Bcl-2 apoptotic switch in cancer development and therapy. Oncogene 2007; 26: 1324-1337.

3 Vogler M, Dinsdale D, Dyer MJ, Cohen GM. Bcl-2 inhibitors: small molecules with a big impact on cancer therapy. Cell Death Differ 2009; 16: 360-367.

4 Hanahan D, Weinberg RA. Hallmarks of cancer: the next generation. Cell 2011; 144: $646-674$

5 Lauria F, Raspadori D, Rondelli D, Ventura MA, Fiacchini M, Visani G et al. High bcl2 expression in acute myeloid leukemia cells correlates with CD34 positivity and complete remission rate. Leukemia 1997; 11: 2075-2078.

6 Davids MS, Letai A. Targeting the B-cell lymphoma/leukemia 2 family in cancer. J Clin Oncol 2012; 30: 3127-3135.

7 Souers AJ, Leverson JD, Boghaert ER, Ackler SL, Catron ND, Chen J et al. ABT-199, a potent and selective $B C L-2$ inhibitor, achieves antitumor activity while sparing platelets. Nat Med 2013; 19: 202-208. 
8 Pan R, Hogdal LJ, Benito JM, Bucci D, Han L, Borthakur G et al. Selective BCL-2 inhibition by ABT-199 causes on-target cell death in acute myeloid leukemia. Cancer Discov 2014; 4: 362-375.

9 Konopleva M, Pollyea DA, Potluri J, Chyla B, Hogdal L, Busman T et al. Efficacy and biological correlates of response in a phase ii study of venetoclax monotherapy in patients with acute myelogenous leukemia. Cancer Discov 2016; 6: 1106-1117.

10 Pullarkat VA, Newman EM. BCL2 inhibition by venetoclax: targeting the Achilles' heel of the acute myeloid leukemia stem cell? Cancer Discov 2016; 6: 1082-1083.

11 Niu X, Zhao J, Ma J, Xie C, Edwards H, Wang G et al. Binding of released bim to mcl-1 is a mechanism of intrinsic resistance to ABT-199 which can be overcome by combination with daunorubicin or cytarabine in AML cells. Clin Cancer Res 2016; 22: 4440-4451.

12 Leverson JD, Zhang H, Chen J, Tahir SK, Phillips DC, Xue J et al. Potent and selective small-molecule MCL-1 inhibitors demonstrate on-target cancer cell killing activity as single agents and in combination with ABT-263 (navitoclax). Cell Death Dis 2015; 6: e1590.

13 Jamil S, Mojtabavi S, Hojabrpour P, Cheah S, Duronio V. An essential role for MCL-1 in ATR-mediated CHK1 phosphorylation. Mol Biol Cell 2008; 19: 3212-3220.

14 Bose $\mathrm{P}$, Grant S. Mcl-1 as a therapeutic target in acute myelogenous leukemia (AML). Leuk Res Rep 2013; 2: 12-14.

15 Nijhawan D, Fang M, Traer E, Zhong Q, Gao W, Du F et al. Elimination of Mcl-1 is required for the initiation of apoptosis following ultraviolet irradiation. Genes Dev 2003; 17: 1475-1486.

16 Cuconati A, Mukherjee C, Perez D, White E. DNA damage response and MCL-1 destruction initiate apoptosis in adenovirus-infected cells. Genes Dev 2003; 17: 2922-2932.

17 Xie C, Edwards H, Caldwell JT, Wang G, Taub JW, Ge Y. Obatoclax potentiates the cytotoxic effect of cytarabine on acute myeloid leukemia cells by enhancing DNA damage. Mol Oncol 2015; 9: 409-421.

18 Niu X, Wang G, Wang Y, Caldwell JT, Edwards $\mathrm{H}$, Xie $\mathrm{C}$ et al. Acute myeloid leukemia cells harboring MLL fusion genes or with the acute promyelocytic leukemia phenotype are sensitive to the Bcl-2-selective inhibitor ABT-199. Leukemia 2014; 28: 1557-1560.

19 Taub JW, Matherly LH, Stout ML, Buck SA, Gurney JG, Ravindranath Y. Enhanced metabolism of 1-beta-D-arabinofuranosylcytosine in Down syndrome cells: a contributing factor to the superior event free survival of Down syndrome children with acute myeloid leukemia. Blood 1996; 87: 3395-3403.

20 Quentmeier H, Zaborski M, Drexler HG. The human bladder carcinoma cell line 5637 constitutively secretes functional cytokines. Leuk Res 1997; 21: 343-350.

21 Qi W, Xie C, Li C, Caldwell JT, Edwards H, Taub JW et al. CHK1 plays a critical role in the anti-leukemic activity of the wee1 inhibitor MK-1775 in acute myeloid leukemia cells. J Hematol Oncol 2014; 7: 53.

22 Ge Y, Dombkowski AA, LaFiura KM, Tatman D, Yedidi RS, Stout ML et al. Differential gene expression, GATA1 target genes, and the chemotherapy sensitivity of Down syndrome megakaryocytic leukemia. Blood 2006; 107: 1570-1581.

23 Ge Y, Stout ML, Tatman DA, Jensen TL, Buck S, Thomas RL et al. GATA1, cytidine deaminase, and the high cure rate of Down syndrome children with acute megakaryocytic leukemia. J Natl Cancer Inst 2005; 97: 226-231.
24 Xie C, Edwards H, Xu X, Zhou H, Buck SA, Stout ML et al. Mechanisms of synergistic antileukemic interactions between valproic acid and cytarabine in pediatric acute myeloid leukemia. Clin Cancer Res 2010; 16: 5499-5510.

25 Edwards H, Xie C, LaFiura KM, Dombkowski AA, Buck SA, Boerner JL et al. RUNX1 regulates phosphoinositide 3-kinase/AKT pathway: role in chemotherapy sensitivity in acute megakaryocytic leukemia. Blood 2009; 114: 2744-2752.

26 Chou TC. Theoretical basis, experimental design, and computerized simulation of synergism and antagonism in drug combination studies. Pharmacol Rev 2006; 58 : 621-681.

27 Xie C, Edwards H, Lograsso SB, Buck SA, Matherly LH, Taub JW et al. Valproic acid synergistically enhances the cytotoxicity of clofarabine in pediatric acute myeloid leukemia cells. Pediatr Blood Cancer 2012; 59: 1245-1251.

28 Xie C, Drenberg C, Edwards H, Caldwell JT, Chen W, Inaba H et al. Panobinostat enhances cytarabine and daunorubicin sensitivities in $\mathrm{AML}$ cells through suppressing the expression of BRCA1, CHK1, and Rad51. PLoS One 2013; 8 : e79106.

29 Aubry JP, Blaecke A, Lecoanet-Henchoz S, Jeannin P, Herbault N, Caron G et al. Annexin $\mathrm{V}$ used for measuring apoptosis in the early events of cellular cytotoxicity. Cytometry 1999; 37: 197-204.

30 Zhao J, Niu X, Li X, Edwards H, Wang G, Wang Y et al. Inhibition of CHK1 enhances cell death induced by the Bcl-2-selective inhibitor ABT-199 in acute myeloid leukemia cells. Oncotarget 2016; 7: 34785-34799.

31 Mah LJ, El-Osta A, Karagiannis TC. gammaH2AX: a sensitive molecular marker of DNA damage and repair. Leukemia 2010; 24: 679-686.

32 Doan PL, Chute JP. The vascular niche: home for normal and malignant hematopoietic stem cells. Leukemia 2012; 26: 54-62.

33 Lagadinou ED, Sach A, Callahan K, Rossi RM, Neering SJ, Minhajuddin M et al. BCL-2 inhibition targets oxidative phosphorylation and selectively eradicates quiescent human leukemia stem cells. Cell Stem Cell 2013; 12: 329-341.

34 Davids MS, Letai A, Brown JR. Overcoming stroma-mediated treatment resistance in chronic lymphocytic leukemia through BCL-2 inhibition. Leuk Lymphoma 2013; 54: $1823-1825$.

35 Choudhary GS, Al-Harbi S, Mazumder S, Hill BT, Smith MR, Bodo J et al. MCL-1 and $\mathrm{BCL}$-xL-dependent resistance to the BCL-2 inhibitor ABT-199 can be overcome by preventing $\mathrm{PI3K} / \mathrm{AKT} / \mathrm{mTOR}$ activation in lymphoid malignancies. Cell Death Dis 2015; 6: e1593.

36 Li L, Pongtornpipat P, Tiutan T, Kendrick SL, Park S, Persky DO et al. Synergistic induction of apoptosis in high-risk DLBCL by BCL2 inhibition with ABT-199 combined with pharmacologic loss of MCL1. Leukemia 2015; 29: 1702-1712.

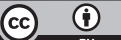

This work is licensed under a Creative Commons Attribution 4.0 International License. The images or other third party material in this article are included in the article's Creative Commons license, unless indicated otherwise in the credit line; if the material is not included under the Creative Commons license, users will need to obtain permission from the license holder to reproduce the material. To view a copy of this license, visit http://creativecommons.org/licenses/ by/4.0/

(c) The Author(s) 2017 